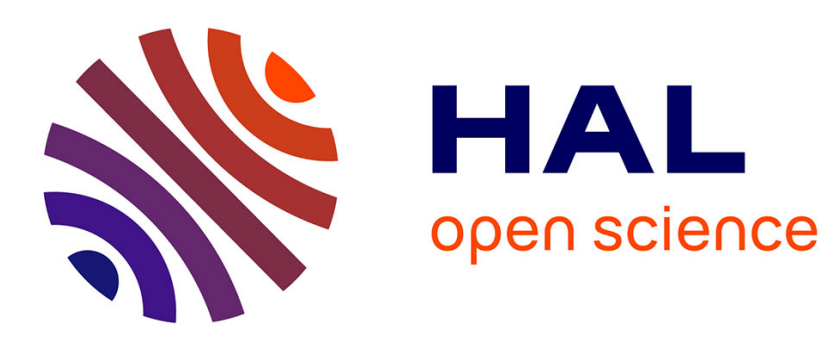

\title{
Mean zonal flow generated by azimuthal harmonic forcing in a rotating cylinder
}

\author{
Alban Sauret
}

\section{To cite this version:}

Alban Sauret. Mean zonal flow generated by azimuthal harmonic forcing in a rotating cylinder. Fluid Dynamics Research, 2015, 47, pp.035506. 10.1088/0169-5983/47/3/035506 . hal-01147831

\section{HAL Id: hal-01147831 \\ https://hal.science/hal-01147831}

Submitted on 1 May 2015

HAL is a multi-disciplinary open access archive for the deposit and dissemination of scientific research documents, whether they are published or not. The documents may come from teaching and research institutions in France or abroad, or from public or private research centers.
L'archive ouverte pluridisciplinaire HAL, est destinée au dépôt et à la diffusion de documents scientifiques de niveau recherche, publiés ou non, émanant des établissements d'enseignement et de recherche français ou étrangers, des laboratoires publics ou privés. 


\title{
Mean zonal flow generated by azimuthal harmonic forcing in a rotating cylinder
}

\author{
Alban Sauret ${ }^{1,2, *}$ \\ ${ }^{1}$ Surface du Verre et Interfaces, UMR 125, 93303 Aubervilliers, France \\ ${ }^{2}$ Department of Mechanical and Aerospace Engineering, Princeton University, Princeton, New \\ Jersey 08544, USA
}

\begin{abstract}
In this paper, we study analytically the flow in a rotating container subjected to an azimuthal forcing. We show that this mechanical forcing generates a correction to the solid body rotation called mean zonal flow, similar to the time oscillation of the rotation rate of an axisymmetric container. This axisymmetric correction induced by nonlinear effects in the Ekman layers modifies the solid body rotation of the fluid in the container. At the leading order, the contribution in the bulk is shown to be an azimuthal flow which scales as the square of the amplitude of the multipolar deformation and is independent of the Ekman number. We also show that the mean zonal flow depends on the symmetry of the angular forcing $\mathrm{n}$ and the ratio of the angular rate of the deformation to the angular rate of the cylinder $\Omega_{R}=\Omega_{\text {orb }} / \Omega_{\text {spin }}$. We found that for an elliptical forcing, $n=2$, the rotation rate of the zonal flow does not depend on the radial position. In addition, the angular rate is found to be asymmetric with respect to $\Omega_{R}$. These scalings are similar to the time harmonic forcing in a cylinder. The particular case of a tidal forcing is also considered.
\end{abstract}

Keywords: Rotating flow, Ekman layers, mechanical forcing, mean zonal flow.

\section{Introduction}

Recent progress in astrophysical measurements have led to a renewed interest in the effects of a harmonic forcing on a rotating fluid. Indeed, harmonic forcings are ubiquitous in planetary bodies which are subject to various type of mechanical forcings due to the presence of other gravitational partners [1, 2]. These harmonic forcings can be time dependent. For instance, a longitudinal libration forcing, corresponds to a time-harmonic oscillation of the rotation rate of a planet [3, 4]. For such a forcing, it has been shown that nonlinear effects in the Ekman layers generate a steady and axisymmetric flow, referred to as the zonal flow. In a cylinder, an analytical description of this steady flow has been obtained by Wang [5] and successfully compared with experimental investigation. It has been shown that the deviation from a solid body rotation scales as the square of the amplitude of libration and is independent of the Ekman number. More recently, the limit of small libration frequency was considered [6] and the zonal flow in presence of inertial waves was studied numerically and experimentally [7, 8, 9]. However, this nonlinear correction is not limited to the cylindrical geometry and is also present in a sphere or spherical shell $[10,11,12]$. This geometry is particularly is relevant for geophysical and astrophysical applications [13]. Many recent studies have focused on this particular forcing as it is simple to implement in numerical

\footnotetext{
*alban.sauret@saint-gobain.com
} 
simulations $[14,9, ?, 15,16]$ and model with an experimental setup [13, 15]. In both the cylindrical and spherical geometries, it has been show that a centrifugal instability induced by the oscillation of the outer container could occur near the outer boundary $[14,9,17]$. However, we do not expect this kind of instability to occur in the system presented in this article.

Other forcings are also present in astrophysical bodies, such as the precession $[18,19]$ or tidal forcings $[20,21,1]$. A planet or satellite is deformed into an elliptical shape by the presence of a gravitational partner. The gravitational field leads to an azimuthal forcing of azimuthal wavenumber $n=2$. Recent experiments [22] and numerical simulation $[23,24]$ have considered a tidal forcing on a rotating sphere and showed that the resonance of inertial waves could lead to a strong zonal flow . In addition, this zonal flow can lead to a shear instability and turbulence [25]. An analytical study devoted to the mean zonal flow in a container elliptically deformed [20,26] provided an analytical description of the nonlinear correction in a tidally deformed sphere that was compared with some experimental results. The study demonstrated that at first order, the base flow has elliptical streamlines [27]. However, the simple model of a sphere subject to a modulation of the tangential velocity with the azimuthal angle has several limitation. For instance, in this case, the fluid does not satisfy the continuity equation on the outer boundary. Finally, in a sphere, the forcing leads to a divergence of the zonal flow near the axis of rotation due to the presence of a critical latitude where the scalings of the Ekman layer no longer holds [28]. It remains unknown whether a multipolar deformation with an arbitrary azimuthal wavenumber leads to the same structure of the nonlinear correction. Moreover, the boundary conditions to apply on a deformed sphere are poorly defined as the deformed shape can lead to a variation in the thickness of the Ekman layer.

In this paper, we aim to show that an azimuthal forcing exhibits the same key features as a temporal forcing. To accommodate a full analytical description without simplification of the shape of the outer boundary, we consider the simpler model composed of two cylindrical plates rotating in a multipolar flow where $n$ is the symmetry of the angular forcing. The particular case $n=2$ corresponds to an elliptical deformation. A schematic of the model geometry is presented in figure 1. Our analytical modeling demonstrates that the azimuthal forcing generates a steady axisymmetric differential rotation in the interior. This nonlinear effect scales as the mean zonal flow driven in a longitudinally librating cylinder. We also characterize the influences of the rotation of the deformation and the influence of the symmetry of the angular forcing, $n$, on the mean zonal flow generated.

\section{Mathematical formulation}

\subsection{Multipolar deformation and generated flow}

We consider the flow in an external cylindrical container of radius filled with an incompressible newtonian fluid of kinematic viscosity. The external container is deformable and rotates around its axis at a constant angular velocity. In addition a multipolar forcing rotating at the angular velocity is applied on the cylinder. The radius of the deformable boundary is given by

We consider the flow in an external cylindrical container of radius $R_{\text {ext }}$ filled with an incompressible Newtonian fluid of kinematic viscosity $\nu$. The external container is deformable and rotates around its

axis at a constant angular velocity $\Omega_{\text {spin }}$. In addition a multipolar forcing rotating at the angular velocity $\Omega_{\text {orb }}$ is applied on the cylinder. The radius of the deformable boundary is given by

$$
R_{e x t}^{d e f}(\theta)=R_{e x t}\left[1+\frac{\beta}{n} \cos (n \theta)\right]+\mathrm{O}\left(\beta^{2}\right),
$$

where $\beta$ and $n$ are the amplitude and the symmetry of the angular forcing, respectively. To model an interaction between a satellite and its planet we generally consider only an elliptical deformation. However, the presence of other satellites can generate deformation with a larger azimuthal wavenumber, 


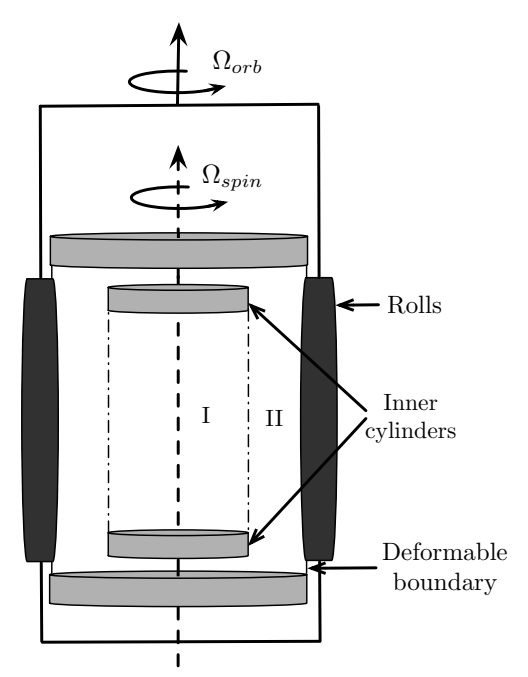

Figure 1: Experimental setup corresponding to the situation described in this paper for $n=2$. A flexible outer cylinder rotating at an angular velocity $\Omega_{\text {spin }}$ is deformed by two rolls rotating at a constant angular velocity $\Omega_{\text {orb }}$. It generates elliptical streamlines in the bulk. Two cylindrical plates rotate at the velocity of the outer cylinder, $\Omega_{\text {spin }}$. We are interested by the zonal flow generated in region I.

leading to a multipolar deformation. The resulting streamfunction can be written

$$
\Psi=-\frac{r^{2}}{2}+\beta \frac{r^{n}}{n} \cos (n \theta)+\mathrm{O}\left(\beta^{2}\right)
$$

with $n \geq 1$. The flow is the superposition of a solid-body rotation and a multipolar strain field [29]. The shape of the streamlines is defined by $\Psi(r, \theta ; \beta)=-k$ where $k$ is a positive constant which is related to the mean radius by $r_{m o y}=\sqrt{2 k}$. The shape of a streamline is further characterized by a local deformation

$$
\beta_{n}=\beta\left(\frac{2 n k}{n-2}\right)^{(n-2) / 2}
$$

that measures its asymmetry. The streamlines for $\beta=0.1$ and varying the symmetry of the angular forcing $n$ are represented in figure 2 . Note that the case $\beta_{n}=\beta$ corresponds to a simple elliptical deformation, $n=2$. In this case, the eccentricity of the streamlines is constant and equal to $\beta_{2}=\beta$. However, for $n \geq 3$, the deformation of the streamlines is no longer homogeneous when the constant $k$ is modified. We notice that for a small value of $k$, i.e. near the center of the flow, the streamlines are quasi-circular. In contrast, for larger $k$ values, further away from the center, the streamlines become more and more deformed and their local eccentricity is defined by equation (3). Note also that for $\beta_{n}>1$ the streamlines become open; thus the analysis will be restricted to the range $0 \leq \beta_{n}<1$.

We define $\Omega_{R}=\Omega_{\text {orb }} / \Omega_{\text {spin }}$ as the ratio of the angular rate of the deformation and the angular rate of the cylinder. To simplify the situation, we consider two disks, separated by an axial distance $H$, and corotating with the cylinder around the $(O z)$-axis at an angular rate equal to the angular rate of the external cylinder. A schematic of the situation is represented in figure 1. A similar system, without the inner disks, has been used in [30] to study the elliptical instability. Experimental measurements have shown that the generated elliptical streamlines are in good agreement with equation (2). Following the analytical study of the flow generated in an oscillating cylinder, we neglect the flow close to the corner. In the two regions indicated in figure 1, I and II, the flow has elliptical streamlines. We are interested in 


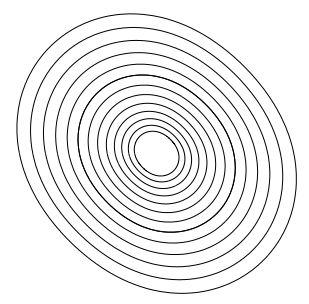

(a)

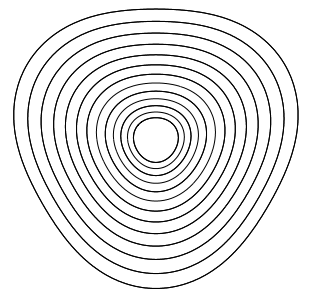

(b)

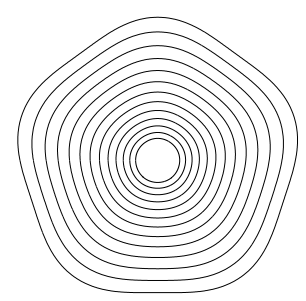

(c)

Figure 2: Streamlines induced by multipolar deformation with $\beta=0.1$ and $r_{\text {moy }}$ ranging between 0.45 and 1 for (a) $n=2$, (b) $n=3$ and (c) $n=5$.

region I, where no- slip boundary conditions must be satisfied for the top and bottom cylinders, which leads to the presence of viscous boundary layers, similar to the situation of a libration-driven flow in a cylinder [5]. The nonlinear flow is different in regions I and II, leading to the presence of Stewartson layers between these two regions, as observed previously in a spherical shell [12,31]. However, for the sake of simplicity, we consider only the flow in region I and do not consider the Stewartson layers that remain localized near the tangent cylinder between the two regions in the asymptotic analysis, provided the forcing is small enough. Indeed, we should emphasize that nonlinearities in the Stewartson layers could lead to instabilities that would broaden the Stewartson layers separating regions I and II and would modify the solution provided in this paper [32, 33, 34, 35]. In addition, for instance for $n=2$, an elliptical instability could develop at a sufficiently large deformation $[30,27,36,1]$. Therefore, our analysis applies fto a sufficiently small forcing amplitude $\beta$ for a given Ekman number $E$.

\subsection{Dimensionless equations}

We define the length scale and time scale of the problem as $R_{\text {ext }}$, the radius of the cylinder and $\Omega_{\text {spin }}{ }^{-1}$, respectively. In the following, we work in the rotating frame of angular velocity $\Omega_{\text {orb }}$. In this frame of reference the flow is stationary and the non-dimensional Navier-Stokes and continuity equations in cylindrical coordinates are given by

$$
\begin{aligned}
u_{r} \frac{\partial u_{r}}{\partial r}+\frac{u_{\theta}}{r} \frac{\partial u_{r}}{\partial \theta}-\frac{u_{\theta}^{2}}{r}+u_{z} \frac{\partial u_{r}}{\partial z}-2 \Omega_{R} u_{\theta} & =-\frac{\partial p}{\partial r}+E \nabla^{2} u_{r} \\
u_{r} \frac{\partial u_{\theta}}{\partial r}+\frac{u_{\theta}}{r} \frac{\partial u_{\theta}}{\partial \theta}+\frac{u_{\theta} u_{r}}{r}+u_{z} \frac{\partial u_{\theta}}{\partial z}+2 \Omega_{R} u_{r} & =-\frac{1}{r} \frac{\partial p}{\partial \theta}+E \nabla^{2} u_{\theta} \\
u_{r} \frac{\partial u_{z}}{\partial r}+\frac{u_{\theta}}{r} \frac{\partial u_{z}}{\partial \theta}+u_{z} \frac{\partial u_{z}}{\partial z} & =-\frac{\partial p}{\partial z}+E \nabla^{2} u_{z} \\
\frac{1}{r} \frac{\partial}{\partial r}\left(r u_{r}\right)+\frac{1}{r} \frac{\partial u_{\theta}}{\partial \theta}+\frac{\partial u_{z}}{\partial z} & =0
\end{aligned}
$$

where $E=\nu /\left(\Omega_{\text {spin }} H^{2}\right)$ is the Ekman number based on the height of the cylinder $H$. Considering a multipolar deformation [30,37], the base flow in the bulk is given by

$$
\begin{aligned}
U_{r} & =-\beta r^{n-1}\left(1-\Omega_{R}\right) \sin (n \theta) \\
U_{\theta} & =r\left(1-\Omega_{R}\right)-\beta r^{n-1}\left(1-\Omega_{R}\right) \cos (n \theta) \\
U_{z} & =0 \\
P & =\frac{r^{2}}{2}\left(1-\Omega_{R}{ }^{2}\right)+\beta \frac{r^{n}}{n}\left[n\left(1-\Omega_{R}\right)-2\right]\left(1-\Omega_{R}\right) \cos (n \theta) .
\end{aligned}
$$


For a fixed deformation $\Omega_{R}=0$ and an elliptical forcing $n=2$, we recover the base flow of a tidal deformation [27]. Note that in the following we will use the notation $\left(U_{r}, U_{\theta}, U_{z}, P\right)$ for the velocity and pressure fields in the bulk and $\left(u_{r}, u_{\theta}, u_{z}, p\right)$ for the velocity and pressure fields in the Ekman layers.

\section{Linear solution in the top and bottom boundary layers}

The velocity and pressure fields (5a)-(5d) are solutions to the Navier?Stokes and continuity equations. However, the velocity is not constant along a streamline; it is lower in the region of large curvature. In addition, at the bottom and top boundaries $(z=[0 ; H])$, the flow must satisfy no-slip boundary conditions. In a rotating frame with the deformation, the no-slip boundary conditions leads to a solid-body rotation at an angular velocity $\Omega_{\text {spin }}-\Omega_{\text {orb }}$. To satisfy these boundary conditions, we need to introduce a viscous layer to match the flow in the bulk to the condition at the solid boundary. For instance, we consider the viscous layer near the bottom boundary, at $z=0$. Because the system is symmetric, we expect the same flow to happen at the top boundary. Let us introduce the boundary layer coordinate:

$$
\zeta=\frac{z}{E^{1 / 2}}
$$

To perform the asymptotic analysis, we assume that the velocity field and the pressure can be expanded in a power series of $\beta$ and $E^{1 / 2}$ :

$$
\begin{gathered}
\mathbf{u}=\mathbf{u}_{0}+\sum_{i=1, j=0} E^{j / 2} \beta^{i} \mathbf{u}_{i}^{j}, \\
p=p_{0}+\sum_{i=1, j=0} E^{j / 2} \beta^{i} p_{i}^{j} .
\end{gathered}
$$

In the following, to simplify the notation, we indicate only the order in $\beta$. Assuming that the radial and orthoradial components of the velocity fields, $u_{r}$ and $u_{\theta}$, are of order $E^{0}$ in the boundary layer, equation (4.d) indicates that the axial velocity is of order $E^{1 / 2}$ smaller in the boundary layer. Thus, in the boundary layers equations (4a)-(4c) can be reduced to the leading order in $E$ and at the order $\beta$ :

$$
\begin{aligned}
\frac{u_{0, \theta}}{r} \frac{\partial u_{1, r}}{\partial \theta}-\frac{2 u_{0, \theta} u_{1, \theta}}{r}-2 \Omega_{R} u_{1, \theta} & =-\frac{\partial p_{1}}{\partial r}+\frac{\partial^{2} u_{1, r}}{\partial \zeta^{2}} \\
u_{1, r} \frac{\partial u_{0, \theta}}{\partial r}+\frac{u_{0, \theta}}{r} \frac{\partial u_{1, \theta}}{\partial \theta}+\frac{u_{0, \theta} u_{1, r}}{r}+2 \Omega_{R} u_{1, r} & =-\frac{1}{r} \frac{\partial p_{1}}{\partial \theta}+\frac{\partial^{2} u_{1, \theta}}{\partial \zeta^{2}} \\
\frac{\partial p_{1}}{\partial \zeta} & =0
\end{aligned}
$$

where $u_{0, \theta}$ is the solution of order 0 in $\beta$. Equation (9c) leads to a constant pressure field in the boundary layer. Its expression is therefore directly given by equation (5d). The symmetry of the multipolar forcing associated with a symmetry of the angular forcing $n$ sets the symmetry of the velocity field

$$
u_{1, r}=\beta \tilde{u}_{1, r} \mathrm{e}^{\mathrm{in} \theta}+\text { c.c } \quad \text { and } \quad u_{1, \theta}=\beta \tilde{u}_{1, \theta} \mathrm{e}^{\mathrm{in} \theta}+\text { c.c. }
$$

where c.c. denotes the complex conjugate. From equations (9a)-(9b) and with the leading-order solution, we obtain the following equations:

$$
\begin{aligned}
\operatorname{in}\left(1-\Omega_{\mathrm{R}}\right) \tilde{\mathrm{u}}_{1, \mathrm{r}}-2 \tilde{\mathrm{u}}_{1, \theta} & =-\frac{\partial \tilde{p}_{1}}{\partial r}+\frac{\partial^{2} \tilde{u}_{1, r}}{\partial \zeta^{2}} \\
2 \tilde{u}_{1, r}+\operatorname{in}\left(1-\Omega_{\mathrm{R}}\right) \tilde{\mathrm{u}}_{1, \theta} & =-\frac{\mathrm{in} \tilde{\mathrm{p}}_{1}}{r}+\frac{\partial^{2} \tilde{u}_{1, \theta}}{\partial \zeta^{2}}
\end{aligned}
$$


For $\zeta=0$, i.e., at the boundary of the cylinder, the velocity field vanishes at the first order in $\beta$. Therefore, we impose the following boundary conditions:

$$
\tilde{u}_{1, r}(\zeta=0)=0, \quad \tilde{u}_{1, \theta}(\zeta=0)=0, \quad \tilde{u}_{1, \zeta}(\zeta=0)=0 .
$$

We note that the velocity field at order $0, \tilde{u}_{0}$, needs to fulfill the no-slip boundary conditions at the upper and lower boundaries. Then the matching condition between the flow in the boundary layer and the flow in the bulk leads to

$$
\lim _{\zeta \rightarrow+\infty} \tilde{u}_{1, r}=-\frac{r^{n-1}\left(1-\Omega_{R}\right)}{2 \mathrm{i}}, \quad \lim _{\zeta \rightarrow+\infty} \tilde{u}_{1, \theta}=-\frac{r^{n-1}\left(1-\Omega_{R}\right)}{2}, \quad \text { and } \lim _{\zeta \rightarrow+\infty} \tilde{u}_{1, \zeta}=0 .
$$

Finally, the solution to this set of equations is given by:

$$
\begin{aligned}
\tilde{u}_{1, r} & =\mathrm{i} \frac{\mathrm{r}^{\mathrm{n}-1}\left(1-\Omega_{\mathrm{R}}\right)}{2}\left(1-\mathrm{e}^{-\lambda \zeta}\right) \\
\tilde{u}_{1, \theta} & =-\frac{r^{n-1}\left(1-\Omega_{R}\right)}{2}\left(1-\mathrm{e}^{-\lambda \zeta}\right) \\
\tilde{u}_{1, z} & =0 \\
\tilde{p}_{1} & ==\frac{r^{n}}{2 n}\left[n\left(1-\Omega_{R}\right)-2\right]\left(1-\Omega_{R}\right)
\end{aligned}
$$

where $\delta$ is the boundary layer thickness,

$$
\lambda=\left(1+s_{ \pm} \mathrm{i}\right)\left|\frac{\mathrm{n}}{2}\left(1-\Omega_{\mathrm{R}}\right)-1\right|^{1 / 2}
$$

with

$$
\begin{array}{ccc}
s_{ \pm}=1 & \text { if } & \frac{n}{2}\left(1-\Omega_{R}\right)-1>0 \\
s_{ \pm}=-1 & \text { if } & \frac{n}{2}\left(1-\Omega_{R}\right)-1<0 .
\end{array}
$$

From expressions (13a)-(13d), we obtain the velocity field and the pressure in the boundary layer:

$$
\begin{aligned}
u_{1, r} & =-r^{n-1}\left(1-\Omega_{R}\right)\left[\sin (n \theta)-\exp (-\delta \zeta) \sin \left(n \theta+s_{ \pm} \delta \zeta\right)\right] \\
u_{1, \theta} & =-r^{n-1}\left(1-\Omega_{R}\right)\left[\cos (n \theta)-\exp (-\delta \zeta) \cos \left(n \theta+s_{ \pm} \delta \zeta\right)\right] \\
u_{1, z} & =0 \\
p_{1} & ==\frac{r^{n}}{n}\left[n\left(1-\Omega_{R}\right)-2\right]\left(1-\Omega_{R}\right) \cos (n \theta)
\end{aligned}
$$

where we have defined

$$
\delta=\left|\frac{n}{2}\left(1-\Omega_{R}\right)-1\right|^{1 / 2}
$$

\section{Non-linear correction}

In the previous section, we showed that the linear response of the flow to a multipolar deformation is a correction at order $\beta$ with harmonics $\mathrm{e}^{\mathrm{in} \theta}$ and $\mathrm{e}^{-\mathrm{in} \theta}$. The nonlinear contribution, i.e., $\beta^{2}$ terms, comes from the nonlinear interactions of the linear flow with itself in the top and bottom Ekman layers and generates harmonics $\mathrm{e}^{2 \mathrm{in} \theta}, \mathrm{e}^{-2 \mathrm{in} \theta}$ and $\mathrm{e}^{\mathrm{i} 0}=1$. This last term referred to as the zonal flow represents a drift of the fluid during the rotation and contributes to the flow after one rotation. 


\subsection{Non linear flow in the bulk}

Contrary to the longitudinal libration forcing where the linear correction is of order $\varepsilon \sqrt{E}$ in the bulk $[5,6,12]$, here we need to consider the linear correction of order $\beta E^{0}$ in the bulk and study whether its nonlinear interaction leads to a mean zonal flow, i.e. to a contribution of harmonics $\mathrm{e}^{\mathrm{i} 0}$. In the bulk at order $\beta^{2}$, the Navier-Stokes equation leads to

$$
\begin{aligned}
r^{2 n-3}(n-1)\left(1-\Omega_{R}^{2}\right)-2 U_{2, \theta} & =-\frac{\partial P_{2}}{\partial r}, \\
U_{2, r} & =0 \\
U_{2, z} & =0
\end{aligned}
$$

Indeed, the nonlinear contribution through the term $\mathbf{U} \cdot \nabla \mathbf{U}$ vanishes and does not generate a nonlinear axisymmetric flow in the absence of top and bottom viscous layers. The physical effects that generate a mean zonal flow in the bulk are thus the same as for a temporal forcing. Actually, (17a)-(17c) indicate that the nonlinear and axisymmetric components of the velocity field only depends on the cylindrical radial coordinate $r$ and can be written:

$$
U_{2, \theta}=r \Omega_{2}(r)
$$

where $\Omega_{2}$ is a function of $r$ that is obtained in the next section.

\subsection{Non linear axisymmetric solution in the boundary layers}

At the order $\beta^{2}$, equations (4a)-(4c) in the boundary layer simplify to

$$
\begin{aligned}
u_{1, r} \frac{\partial u_{1, r}}{\partial r}+\frac{u_{1, \theta}}{r} \frac{\partial u_{1, r}}{\partial \theta}-\frac{u_{1, \theta}^{2}}{r}-2 u_{2, \theta} & =-\frac{\partial p_{2}}{\partial r}+\frac{\partial^{2} u_{2, r}}{\partial \zeta^{2}} \\
u_{1, r} \frac{\partial u_{1, \theta}}{\partial r}+\frac{u_{1, \theta}}{r} \frac{\partial u_{1, \theta}}{\partial \theta}+\frac{u_{1, \theta} u_{1, r}}{r}+2 u_{2, r} & =\frac{\partial^{2} u_{2, \theta}}{\partial \zeta^{2}} \\
\frac{\partial p}{\partial \zeta} & =0
\end{aligned}
$$

Substituting expressions (10a)?(10b) into equation (19a)?(19c), we obtain

$$
\begin{aligned}
\frac{\partial^{2} u_{2, r}}{\partial \zeta^{2}}+2 u_{2, \theta} & =\frac{\partial p_{2}}{\partial r}+r^{2 n-3}(n-1)\left(1-\Omega_{R}\right)^{2}\left(1-\mathrm{e}^{-\lambda \zeta}\right)\left(1-\mathrm{e}^{-\lambda^{*} \zeta}\right) \\
\frac{\partial^{2} u_{2, \theta}}{\partial \zeta^{2}}-2 u_{2, r} & =0
\end{aligned}
$$

This leads to a differential equation in $\zeta$ for $\left(u_{2, r}+\mathrm{i} \mathrm{u}_{2, \theta}\right)$ :

$$
\begin{array}{r}
\left(\frac{\partial^{3}}{\partial \zeta^{3}}-2 \mathrm{i} \frac{\partial}{\partial \zeta}\right)\left(u_{2, r}+\mathrm{i} \mathrm{u}_{2, \theta}\right)=r^{2 n-3}(n-1)\left(1-\Omega_{R}\right)^{2}\left(\lambda \mathrm{e}^{-\lambda \zeta}\right. \\
\left.+\lambda^{*} \mathrm{e}^{-\lambda^{*} \zeta}-\left(\lambda+\lambda^{*}\right) \mathrm{e}^{-\left(\lambda+\lambda^{*}\right) \zeta}\right)
\end{array}
$$

We write the no-slip conditions on the bottom boundary, i.e., for $\zeta=0$,

$$
u_{2, r}(\zeta=0)=0 \quad \text { and } \quad u_{2, \theta}(\zeta=0)=0
$$

Morevoer, the solutions in the Ekman layer and in the bulk need to match and leads to

$$
\lim _{\zeta \rightarrow+\infty} u_{2, r}=0 \quad \text { and } \quad \lim _{\zeta \rightarrow+\infty} u_{2, \theta}=\operatorname{ir} \Omega_{2}(\mathrm{r})
$$


Therefore, the solution to equations (21)-(23) is

$$
\begin{aligned}
u_{2, r}+\mathrm{iu}_{2, \theta}= & r^{2 n-3}(n-1)\left(1-\Omega_{R}\right)^{2}\left[\frac{\mathrm{e}^{-\kappa \zeta}-\mathrm{e}^{-\left(\lambda+\lambda^{*}\right) \zeta}}{\kappa^{2}-\left(\lambda+\lambda^{*}\right)^{2}}-\frac{\mathrm{e}^{-\kappa \zeta}-\mathrm{e}^{-\lambda \zeta}}{\kappa^{2}-\lambda^{2}}\right. \\
& \left.-\frac{\mathrm{e}^{-\kappa \zeta}-\mathrm{e}^{-\lambda^{*} \zeta}}{\kappa^{2}-\lambda^{* 2}}\right]+\mathrm{ir} \Omega_{2}(\mathrm{r})\left(1-\mathrm{e}^{-\kappa \zeta}\right)
\end{aligned}
$$

where $\kappa=1+\mathrm{i}$. The continuity equation in the boundary layer for the nonlinear and axisymmetric flow, $\beta^{2} \mathrm{e}^{\mathrm{i} 0}$, is

$$
\frac{\partial}{\partial r}\left(r u_{2, r}\right)+\frac{1}{\sqrt{E}} \frac{\partial u_{2, z}}{\partial \zeta}=0
$$

Because $U_{2, r}=0$ and $U_{2, \theta}$ is a function of $r$ only, the continuity equation in the bulk implies that the axial velocity does not depend on the axial coordinate $z$ :

$$
\frac{\partial U_{2, z}}{\partial z}=0
$$

Moreover, the system exhibits an equatorial symmetry. Therefore, we have for $z=H / 2, U_{z, 2}=0$. As a consequence, in the bulk, $U_{2, z}=0$. Thus, to satisfy the matching condition in the bulk, the equation (25) implies that $u_{2, r}$ satisfies the relation

$$
\lim _{\zeta \rightarrow+\infty} u_{2, z}=-\sqrt{E} \int_{0}^{+\infty} \frac{\partial}{\partial r}\left(r u_{2, r}\right) \mathrm{d} \eta=0
$$

Let us substitute the solution (24) into the relation (27). We then obtain a differential equation for $\Omega_{2}(r)$ with $S>0$ :

$$
\begin{aligned}
& \frac{r^{2}}{2} \frac{\mathrm{d} \Omega_{2}(r)}{\mathrm{d} r}+r \Omega_{2}(r) \\
& +\frac{r^{2 n-3}(n-1)^{2}\left(1-\Omega_{R}\right)^{2}[1-S[2+S(10 \sqrt{S}-2 S-9)]]}{2\left(4 S^{2}+1\right)\left(S^{2}-1\right)}=0
\end{aligned}
$$

where $S$ is defined by

$$
S=\left|\frac{n}{2}\left(1-\Omega_{R}\right)-1\right|
$$

The constant of integration has to be taken equal to zero to avoid the divergence of $\Omega_{2}(r)$ at $r=0$. Finally, the solution is given by

$$
\Omega_{2}=-\frac{\left.r^{2 n-4}(n-1)^{2}\left(1-\Omega_{R}\right)^{2}[1-S[2+S(10 \sqrt{S}-2 S-9))]\right]}{2\left(4 S^{2}+1\right)\left(S^{2}-1\right)}
$$

\section{Discussion}

From expression (30), we see an important difference between an elliptical forcing, $n=2$, and a higher order azimuthal forcing, $n>2$. For $n=2$, the structure of the rotation rate associated with the mean zonal flow is the same as for a longitudinal libration forcing [5]: the rotation rate does not depend on the radius. In contrast, for larger values of $n, \Omega_{2}$ depends on the radial position in the flow. Figure 3 shows the radial dependence of the angular rotation rate $\Omega_{2}$, associated with the zonal flow for,$=2$. The mean zonal flow vanishes for $\Omega_{R}=1$, i.e., at an angular rate of the deformation equal to the angular rate of the cylinder. Here, the flow is only a solid body rotation in the bulk and no pumping is generated in the top and bottom Ekman layers. Note also a singular point for $\Omega_{R}=1-2 / n$, for example, $\Omega_{R}=0$ for an elliptical deformation, $n=2$. In this situation, $S=0$ and the Ekman layer becomes singular which is 


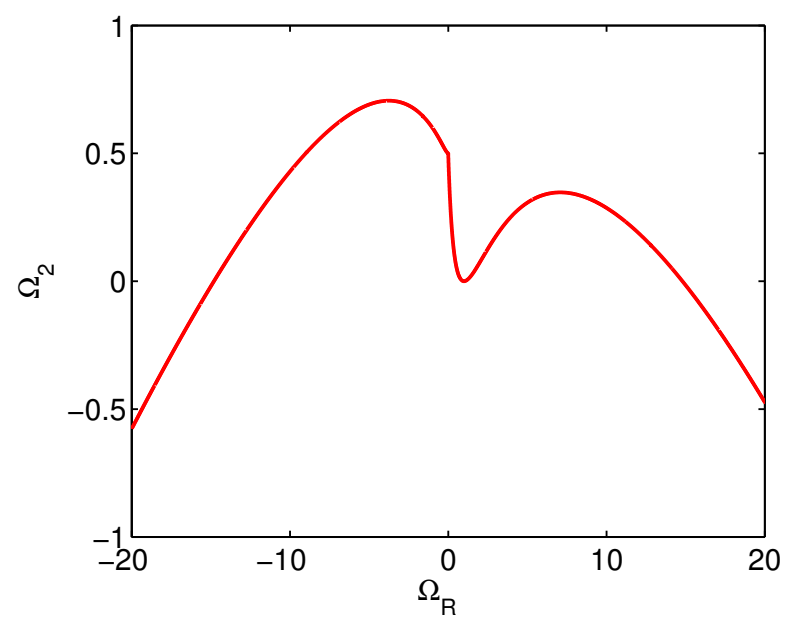

Figure 3: Angular rate associated with the mean zonal flow generated by an elliptical forcing, $n=2$ in the cylinder (solid red line) as a function of $\Omega_{R}$.

beyond the calculation presented in this paper. Therefore, the present analytical solution cannot be used anymore. However, we note that the plot shown in figure 3 can be smoothed by continuity around this critical value, as observed in an oscillating cylinder [5]. The correction can also be cyclonic or anticyclonic as it is positive for

$$
\frac{n-2}{n}-\frac{2\left[1+2^{1 / 3}+2^{2 / 3}\right]}{n}<\Omega_{R}<\frac{n-2}{n}+\frac{2\left[1+2^{1 / 3}+2^{2 / 3}\right]}{n}
$$

and decreases to $-\infty$ for a large value of $\Omega_{R}$.

Figure 4 shows the mean rotation rate $\Omega_{2}$ for a given value of $\Omega_{R}=2$ and various values of the azimuthal wavenumber of the perturbation. As mentioned previously, the elliptical forcing is the only case where the angular velocity associated to the mean zonal flow does not depend on the radius. Increasing the azimuthal wavenumber of the forcing leads to a flattening of the angular rate near the axis of rotation and an increase in the value at $r=1$. This result is in agreement with the definition of the local curvature (3) given in the introduction of the article.

We should also mention that owing to the deviation from the solid-body rotation between the two cylindrical plates, the axisymmetric flow in the interior (for $r<1$ ) does not rotate at the same velocity as in the outer region $(r>1)$. Therefore, this discontinuity will be smooth in Stewartson layer exhibiting the same structure as in two cylindrical plates rotating at different velocities [38,31]. The presence of such layers has already been described for a time-harmonic forcing in a rotating shell [12], where it was in rather good agreement with numerical simulations.

We have considered the flow in the inner region I, but we should notice that in the region II (see figure 1 ), the elliptical streamlines also induced a correction near the external boundary at $r=R_{\text {ext }}$. In the 


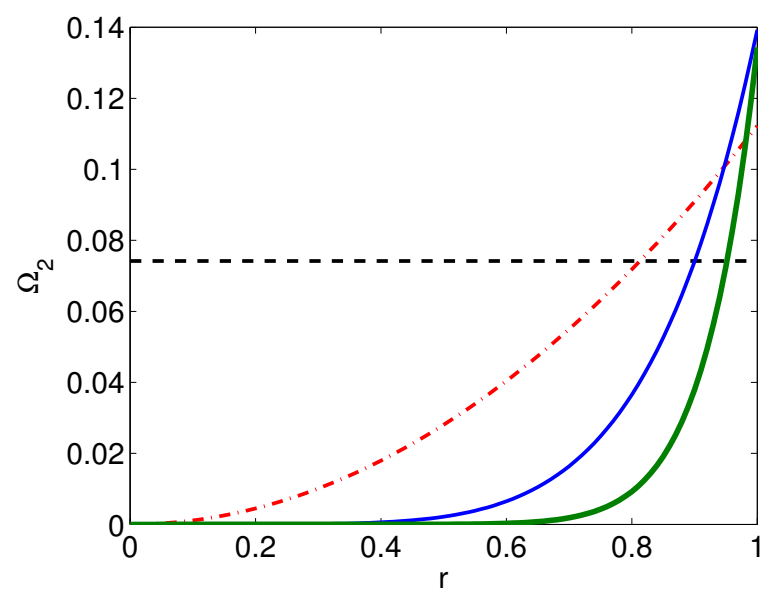

Figure 4: Angular rate associated with the mean zonal flow generated by various multipolar forcings in the cylinder for $\Omega_{R}=2$. The dashed black line is an elliptical forcing, $n=2$; the dashed-dotted red line is a tripolar forcing, $n=3$; solid blue line corresponds to $n=5$, and the solid thick green line is $n=8$.

limit of the small deformations, $\beta \ll 1$, the correction at the first order in $\beta$ is [39]:

$$
\begin{aligned}
U_{r}=-\beta r^{n-1}\left(1-\Omega_{R}\right) \sin (n \theta)-\left(1-\Omega_{R}\right) & \frac{\beta \sqrt{E}(1-n)}{\sqrt{n}} \mathrm{e}^{k_{\nu}\left(r-R_{e x t}\right)} \\
& \times \sin \left(k_{\nu}\left(r-R_{\text {ext }}\right)+n \theta+\frac{\pi}{4}\right), \\
U_{\theta}=r\left(1-\Omega_{R}\right)-\beta r^{n-1}\left(1-\Omega_{R}\right) \cos (n \theta)-\left(1-\Omega_{R}\right) \frac{\beta k_{\nu} \sqrt{E}(1-n)}{n^{3 / 2}} \mathrm{e}^{k_{\nu}\left(r-R_{e x t}\right)} & \times\left[\cos \left(k_{\nu}\left(r-R_{\text {ext }}\right)+n \theta+\frac{\pi}{4}\right)-\sin \left(k_{\nu}\left(r-R_{\text {ext }}\right)+n \theta+\frac{\pi}{4}\right)\right], \\
U_{z}=0, &
\end{aligned}
$$

where we defined

$$
k_{n}=\sqrt{\frac{n}{2 E}} .
$$

In this paper, we have demonstrated the similarity of the mean zonal flows generated by a time harmonic and an azimuthal forcing between two cylindrical plates. However, in addition to the mean zonal flow, inertial waves are also susceptible to appear, depending on the frequency of perturbation. In a cylinder driven by longitudinal libration at the frequency $\omega$ of its outer boundary, the emitted inertial waves satisfy the dispersion relation $\omega=2 \sin \theta$ where $\theta$ is the angle between the direction of propagation of the inertial waves and the axis of rotation [40, 41]. For a multipolar forcing, we expect to see the propagation of inertial waves in the range

$$
n-2<\omega<n+2
$$

To conclude, we have demonstrated the analogy between time and azimuthal forcings in a rotating cylinder. We note that the same analogy is expected to be present in a rotating sphere or shell. However, in this geometry, the boundary conditions are more complicated to apply and the presence of a critical latitude may lead to a divergence of the flow at some particular point $[11,28]$. In addition, the longitudinal libration of an ellipsoid is of importance for geophysical and astrophysical applications and will also need to be considered $[42,43,44]$. This paper therefore constitutes a first step toward understanding the 
effects of both time harmonic and azimuthal forcings on the mean zonal flow in ellipsoidal containers and astrophysical bodies.

Acknowledgments: the author thanks S. Le Dizès, M. Le Bars and D. Cébron for helpful discussions.

\section{References}

[1] M. Le Bars, D. Cébron, and P. Le Gal, "Flows driven by libration, precession, and tides," Ann. Rev. Fluid Mech., vol. 47, pp. 163-193, 2015.

[2] D. Cébron, M. Le Bars, P. Le Gal, C. Moutou, J. Leconte, and A. Sauret, "Elliptical instability in hot jupiter systems," Icarus, vol. 226, p. 1642?1653, 2013.

[3] R. L. Comstock and B. G. Bills, "A solar system survey of forced librations in longitude," Journal of Geophysal Research, vol. 108, p. 113, 2003.

[4] N. Rambaux, T. V. Hoolst, and O. Karatekin, "Librational response of Europa, Ganymede, and Callisto with an ocean for non-keplerian orbit," $A \& E$ A, vol. 527, p. A118, 2011.

[5] C.-Y. Wang, "Cylindrical tank of fluid oscillating about a state of steady rotation," J. Fluid Mech., vol. 41, pp. 581-592, 1970.

[6] F. H. Busse, "Zonal flow induced by longitudinal librations of a rotating cylindrical cavity," Physica D: Nonlinear Phenomena, vol. 240, pp. 208-211, 2011.

[7] J. Noir, M. A. Calkins, M. Lasbleis, J. Cantwell, and J. M. Aurnou, "Experimental study of librationdriven zonal flows in a straight cylinder," Physics of the Earth and Planetary Interiors, vol. 182, pp. 98-1106, 2010.

[8] J. M. Lopez and F. Marques, "Instabilities and inertial waves generated in a librating cylinder," $J$. Fluid Mech., vol. 687, pp. 171-193, 2011.

[9] A. Sauret, D. Cébron, M. Le Bars, and S. Le Dizès, "Fluid flows in librating cylinder," Phys.Fluids, vol. 24, p. 026603, 2012.

[10] K. D. Aldridge, An experimental study of axisymmetric inertial oscillations of a rotating liquid sphere. $\mathrm{PhD}$ thesis, Massachusetts Institute of Technology, 1967.

[11] F. Busse, "Mean zonal flows generated by librations of a rotating spherical cavity," J. Fluid Mech., vol. 650, pp. 505-512, 2010.

[12] A. Sauret and S. Le Dizès, "Libration-induced mean flow in a spherical shell," J. Fluid Mech, vol. 718, pp. 181-209, 2013.

[13] J. Noir, F. Hemmerlin, J. Wicht, S. Baca, and J. M. Aurnou, "An experimental and numerical study of librationally driven flow in planetary cores and subsurface oceans," Physics of the Earth and Planetary Interiors, vol. 173, pp. 141-152, 2009.

[14] M. A. Calkins, J. Noir, J. Eldredge, and J. M. Aurnou, "Axisymmetric simulations of libration-driven fluid dynamics in a spherical shell geometry," Physics of Fluids, vol. 22, pp. 1-12, 2010.

[15] A. Sauret, D. Cebron, C. Morize, and M. Le Bars, "Experimental and numerical study of mean zonal flows generated by librations of a rotating spherical cavity," J. Fluid Mech., vol. 662, pp. 260-268, 2010 . 
[16] A. Sauret, D. Cébron, and M. Le Bars, "Spontaneous generation of inertial waves from boundary turbulence in a librating sphere," J. Fluid Mech., vol. 728, p. R5, 2013.

[17] S. Koch, U. Harlander, C. Egbers, and R. Hollerbach, "Inertial waves in a spherical shell induced by librations of the inner sphere: experimental and numerical results," Fluid Dyn. Res., vol. 45, p. 035504, 2013.

[18] F. H. Busse, "Steady fluid flow in a precessing spheroidal shell," J. Fluid Mech., vol. 33, pp. 739-751, 1968.

[19] W. V. R. Malkus, "Precession of the earth as the cause of geomagnetism," Science, vol. 160, pp. 259$264,1968$.

[20] S. T. Suess, "Viscous flow in a deformable rotating container," J. Fluid Mech., vol. 45, pp. 189-201, 1971.

[21] W. V. R. Malkus, "An experimental study of global instabilities due to tidal (elliptical) distortion of a rotating elastic cylinder," Geophys. Astrophys. Fluid Dyn., vol. 48, p. 123, 1989.

[22] C. Morize, M. Le Bars, P. Le Gal, , and A. Tilgner, "Experimental determination of zonal winds driven by tides," Phys. Rev. Lett., vol. 104, p. 214501, 2010.

[23] A. Tilgner, "Zonal wind driven by inertial modes," Phys. Rev. Lett., vol. 99, p. 194501, 2007.

[24] B. Favier, A. J. Barker, C. Baruteau, and G. I. Ogilvie, "Non-linear evolution of tidally forced inertial waves in rotating fluid bodies," Mon. Not. Roy. Astron. Soc., vol. 439, pp. 845-860, 2014.

[25] A. Sauret, Le Bars, M., and P. Le Gal, "Tide-driven shear instability in planetary liquid cores," Geophysical Research Letters, vol. 41, pp. 6078-6083, 2014.

[26] S. T. Suess, Some effects of gravitational tides on a rotating fluid. PhD thesis, University of California, 1970.

[27] R. R. Kerswell, "Elliptical instability," Annual Review of Fluid Mechanics, vol. 34, pp. 83-113, 2002.

[28] S. Kida, "Steady flow in a rapidly rotating sphere with weak precession," Journal of Fluid Mechanics, vol. 680, pp. 150-193, 2011.

[29] S. Le Dizès and C. Eloy, "Short-wavelength instability of a vortex in a multipolar strain field," Phys. Fluids, vol. 11, pp. 500-502, 1999.

[30] C. Eloy, P. Le Gal, and S. Le Dizès, "Experimental study of the multipolar vortex instability," Phys. Rev. Lett., vol. 85, pp. 3400-3403, 2000.

[31] K. Stewartson, "On almost rigid rotations. part 2," J. Fluid Mech., vol. 26, pp. 131-144, 1966.

[32] W. G. Fruh and P. L. Read, "Experiments on a barotropic rotating shear layer. part 1. instability and steady vortices," J. Fluid Mech., vol. 383, pp. 143-173, 1999.

[33] R. Hollerbach, "Instabilities of the stewartson layer part 1. the dependence on the sign of ro," J. Fluid Mech., vol. 492, pp. 289-302, 2003.

[34] R. Hollerbach, B. Futterer, T. More, and C. Egbers, "Instabilities of the stewartson layer part 2. supercritical mode transitions," Theor. Comp. Fluid Dyn., vol. 18, pp. 197-204, 2004.

[35] N. Schaeffer and P. Cardin, "Quasigeostrophic model of the instabilities of the stewartson layer in flat and depth-varying containers," Phys. Fluids, vol. 17, p. 104111, 2005. 
[36] C. Eloy, P. Le Gal, and S. Le Dizès, "Elliptic and triangular instabilities in rotating cylinders," J. Fluid Mech., vol. 476, pp. 357-388, 2003.

[37] S. Le Dizès, "Three-dimensional instability of a multipolar vortex in a rotating flow," Phys. Fluids, vol. 12 , pp. 2762-2774, 2000.

[38] K. Stewartson, "On almost rigid rotations," J. Fluid Mech., vol. 3, pp. 17-26, 1957.

[39] C. Eloy, Instablité multipolaire de tourbillons. PhD thesis, Université Aix-Marseille II, Institut de Recherche sur les Phnomnes Hors Equilibre, 2000.

[40] H. P. Greenspan, The Theory of rotating fluids. Cambridge University Press, Cambridge, 1968.

[41] K. Zhang, P. Earnshaw, X. Liao, and F. H. Busse, "On inertial waves in a rotating fluid sphere," Journal of Fluid Mechanics, vol. 437, pp. 103-119, 2001.

[42] J. Noir, D. Cébron, M. Le Bars, A. Sauret, and J. M. Aurnou, "Experimental study of librationdriven zonal flows in non-axisymmetric containers," Physics of the Earth and Planetary Interiors, vol. 204-205, pp. 1-10, 2012.

[43] K. Zhang, K. H. Chan, and X. Liao, "On fluid motion in librating ellipsoids with moderate equatorial eccentricity," Journal of Fluid Mechanics, vol. 673, pp. 468-479, 2011.

[44] K. H. Chan, X. Liao, and K. K. Zhang, "Simulations of fluid motion in ellipsoidal planetary cores driven by longitudinal libration," Physics of the Earth and Planetary Interiors, vol. 187, pp. 391-403, 2011. 